\title{
A ROBOTIZED NEEDLE INSERTION DEVICE FOR PERCUTANEOUS PROCEDURES
}

\author{
Olivier Piccin, Pierre Renaud \\ LICIA(EA3434), INSA-Strasbourg \\ 24, Bd de la Victoire, 67084 Strasbourg, FRANCE \\ Email: Olivier.Piccineinsa-strasbourg.fr
}

\author{
Laurent Barbé, Bernard Bayle \\ Benjamin Maurin, Michel de Mathelin \\ LSIIT (UMR CNRS-ULP 7005), Strasbourg I University \\ Bd. S. Brant, BP 10413, 67412 Illkirch, FRANCE \\ Email: barbedeavr.u-strasbg.fr
}

\begin{abstract}
In this paper, a new robotized needle insertion device is proposed for computer-assisted percutaneous therapy. The insertion device is integrated in a robotic system dedicated to gesture guidance in a Computed Tomography (CT) scan. The presented design fulfills the stringent requirements of such a medical application: compatibility with a CT-scan and haptic control by the practitioner are ensured as well as safety and sterilization. The novel design of the insertion device is first presented, outlining its main properties, before introducing preliminary experimental results.
\end{abstract}

\section{INTRODUCTION}

Computed Tomography (CT) is an imaging technique which is now widely used for medical diagnosis as well as interventions. Minimally invasive percutaneous procedures performed with a CT-scan for chest or abdomen treatments [1] reduce the trauma for the patient while ensuring the accuracy of the surgeon's gesture. In such treatment however, the radiologist is exposed to harmful X-rays during needle insertion. X-rays intensity is even more harmful when CT fluoroscopy-guided intervention is achieved to improve gesture accuracy. A robotic system designed to position and introduce the needle at a safer distance can therefore be an adequate solution to improve percutaneous procedures.

CT-guided surgery systems have already been proposed. The RCM+PAKY system [2-4] has been efficiently used in the operating room. Clinical experiments $[5,6]$ have also been achieved in other medical fields to validate computer-aided surgery by needle insertion. In these systems, image-guided positioning was resorted to in spite of two noticeable downsides. First, the surgeon receives no haptic feedback, when his/her gesture needs to take the insertion force into account and adapt accordingly. Second, the effects of chest motion due to patient breathing have not been considered at any time, although their importance is crucial for chest or abdomen applications.

To solve these problems, we are currently developing a robotized CT-guided needle insertion system with haptic feedback [7]: a needle positioning robot which is compatible with the CT-scan has been developed [8] and can be fastened to the patient so as to compensate for chest motion due to patient breathing. The needle positioning robot is based on a 5-DOF parallel structure to set the position and orientation of the needle. A stereotactic system allows to locate the target of the treatment in reference with the robot using only one CT-scan image [9]. The stiffness of the structure is improved thanks to its parallel architecture, and compactness is optimized so that a standard CT-scan can be used for the intervention.

As far as we know, very few papers have been published about the design of a robotized needle insertion device to control the penetration of the needle. Friction-based insertion devices have been proposed in $[6,10]$. They are mostly interesting because of the large stroke of the needle: since its displacement is obtained by a continuous rotation movement, the system compactness is ensured. Friction also mechanically allows to limit the insertion force, since those devices provide the surgeon with no haptic feedback. On the contrary, in the case of our robotic 
system the needle displacement and the interaction forces have to be evaluated to give the surgeon the needle tip position as well as the insertion effort. In this paper, a new robotized needle insertion device is proposed to fulfill the requirements of remote force-controlled needle insertion. The design constraints which have to be complied with include compactness, as the device has to be CT-scan compatible, and preservation of a safe and sterile environment owing to the medical context involved.

In the next section, the overall structure of the needle positioning robot is briefly reviewed. The design of the proposed needle insertion device shall then be presented, outlining its kinematic properties and its thorough adequacy with medical requirements. In the last section, the current development of the prototype shall be described and a first validation of the proposed architecture will be demonstrated through preliminary experimental results. Conclusions on further developments of the insertion device and the overall robotized system will finally be drawn.

\section{THE ROBOTIZED PERCUTANEOUS THERAPY SCE- NARIO}

In this section, the overall structure of the needle positioning robot is briefly recalled, with the associated intervention procedure. It outlines the design requirements of the needle insertion device. Further details may be found in $[7,8]$.

\section{The Robotic Positioning Device}

The needle positioning robot called CT-Bot is a parallel mechanism attached to the abdomen of the patient with special straps. Its volume is less than $200 \times 200 \times 250 \mathrm{~mm}^{3}$. It weighs less than $2.5 \mathrm{~kg}$ which is suitable for most patients. This mechanism features the five degrees of freedom required to position the needle and orient the line supporting its axis. Figures 1 and 2 show the CAD model of the CT-Bot and a constructed prototype.

The structure is composed of one 6-bar linkage and a 4-bar linkage joined together by the end-effector. Due to the choice of the legs geometry, the forward and inverse kinematic models can be computed analytically.

The positioning of the end-effector is obtained using ultrasonic motors. The high holding torque of these motors fits particularly the safety requirements in this medical application: the needle positioning robot remains motionless in case of accidental power failure.

\section{The Robotized Percutaneous Procedure}

Once a percutaneous therapy is decided by the practitioner, the procedure is composed of three main steps:

\section{1 - Registration of the Robot with Respect to the}

Patient. The robot registration in the CT-scan is performed

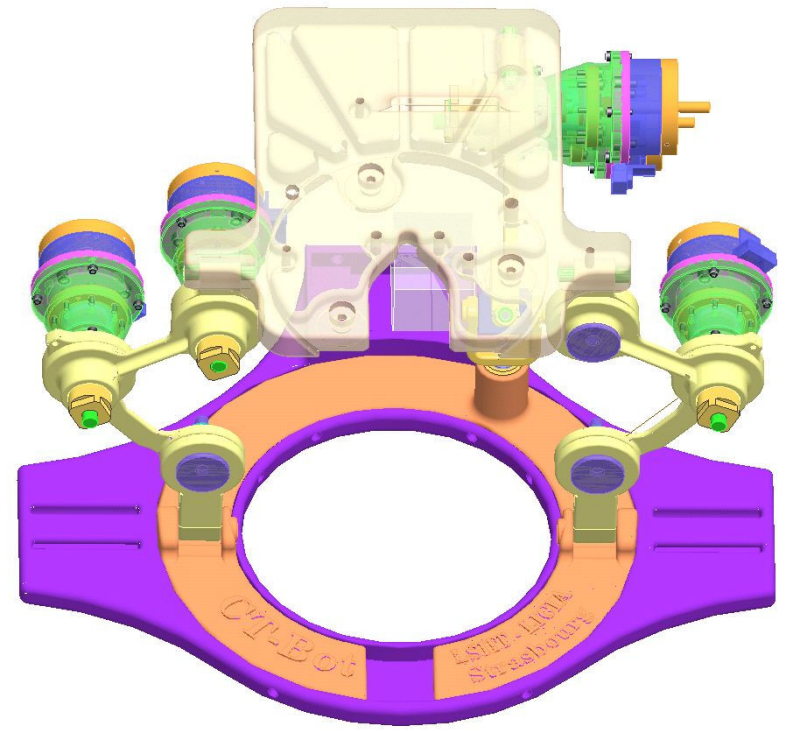

Figure 1. CT-BOT CAD MODEL.

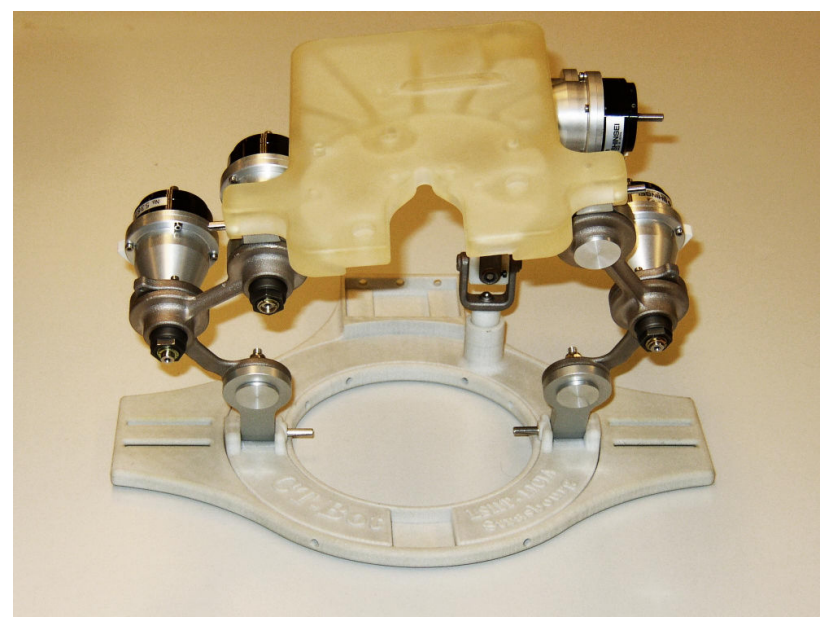

Figure 2. CT-BOT PROTOTYPE.

using a specifically developed marker linked to the base of the robot. From one CT-scan image of this marker, the robot base position and orientation can be computed [9]. It is necessary to notice that, due to the limited thickness of the CT-scan image slices, the needle tip is not always visible. In particular, the needle tip is very likely to be invisible in the slice of the target (e.g. a tumor). It is therefore necessary to estimate the needle tip position with respect to the CT-scan images using the needle insertion device.

2 - Displacement of the Needle Positioning Robot. From the previous registration, the robot end-effector can be 
positioned for the insertion procedure. The positioning is performed using a collision avoidance algorithm so that the device configuration is always valid.

\section{3 - Force-Controlled Needle Insertion Using Haptic} Feedback. When the robot end-effector position and orientation are obtained, the needle insertion can be performed. The practitioner uses the visual feedback of the CT-scan image to estimate the required displacement and the haptic feedback to get information on the nature of the tissues. The self-rotation of the needle may also be needed to orient the bevel of the needle.

The needles have dimensions which vary from 100 to 180 $\mathrm{mm}$ in length and 0.5 to $2.4 \mathrm{~mm}$ in diameter. The available space to carry out insertion is limited by the size of the ring of the scanner and the corpulence of the patient. The minimal available volume corresponds to a $200 \mathrm{~mm}$ radius half-sphere centered on the entry point of the needle on the patient's skin. A main constraint of the insertion device is therefore its compactness.

To summarize, in this robotized percutaneous therapy scenario, the needle insertion device has to provide:

1. two degrees-of-freedom, the needle insertion displacement and its self-rotation,

2. the insertion force measurement to provide the practitioner with a force feedback,

3. an insertion principle compatible with the small available volume in the CT-scan, and which allows us also to locate the needle tip with respect to the robot end-effector,

4. safety and sterilization properties.

\section{DESIGN OF THE INSERTION DEVICE}

In this section, the main elements of the insertion device are presented to outline its integration on the existing needle positioning robot. The device design is then detailed, before introducing the needle kinematics, the insertion force measurement principle and the device compatibility with medical requirements.

\section{Global Overview}

The main components of the needle insertion device are presented on Figure 3 with the platform of the needle positioning robot (item 1 on the figure). The device is composed of three parts: the interface supporting the sensors for needle insertion force measurement (item 2), the driving mechanism including the actuators for all the needle displacements (item 3 ) and the needle insertion mechanism that performs both grasp and displacements of the needle (item 4).

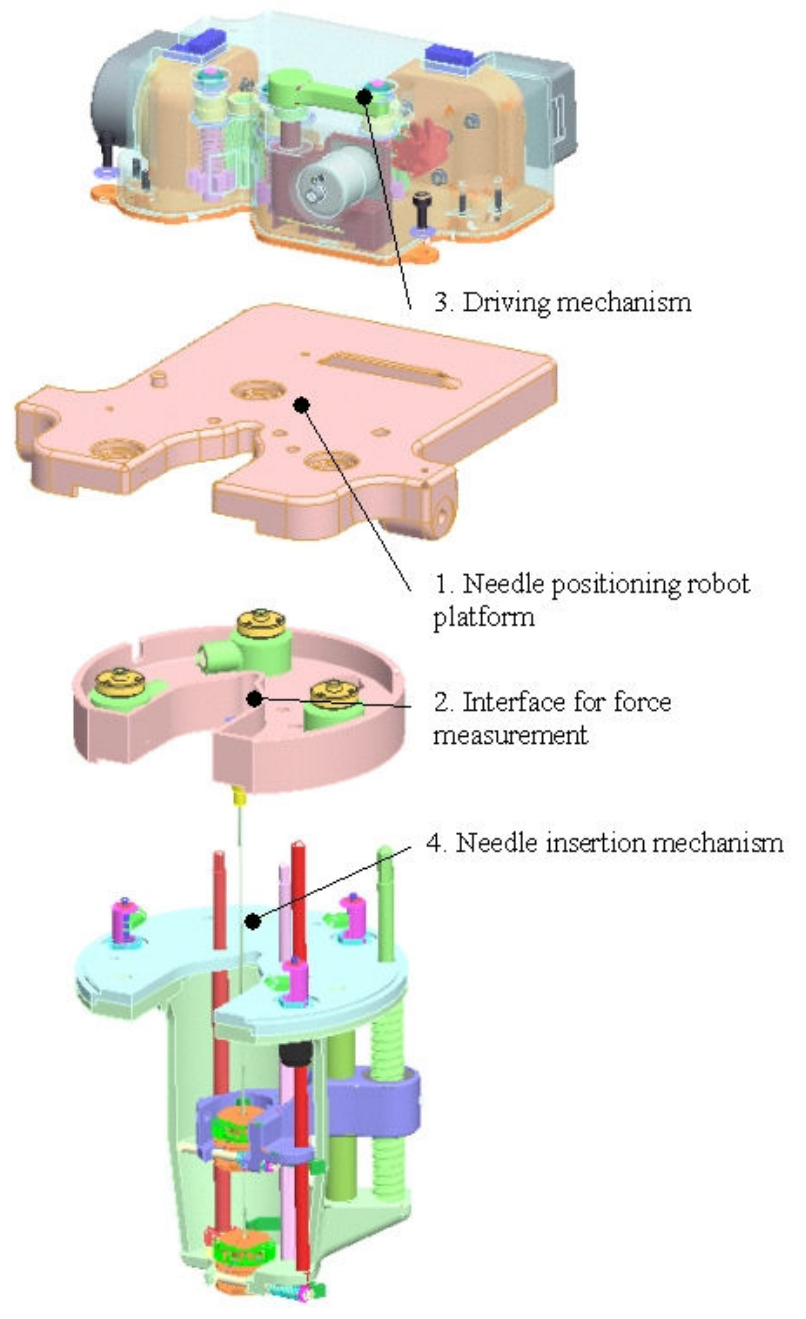

Figure 3. EXPLODED VIEW OF THE NEEDLE INSERTION DEVICE.

\section{Design presentation}

Grasping of the Needle. The grasping of the needle is carried out using two specifically-designed miniature chucks allowing the insertion device to grasp needles with various diameters. Each chuck is composed of a body, three jaws, a spur gear and an indexing ring (Figure 4). The rotation of the gear with respect to the body generates a radial displacement of the jaws and consequently the grasp or release of the needle.

Insertion Mechanism Design. The insertion mechanism is composed of a casing and a mobile carriage in translation with respect to the casing (Figure 5). A chuck $C_{1}$ is mounted on the casing while the other $C_{2}$ is linked to the carriage. This latter is displaced by means of the shaft 1 thanks to a nut. The tightening/release of the chucks $C_{1}$ and $C_{2}$ is obtained by the rotation of the shafts 2 and 3. 


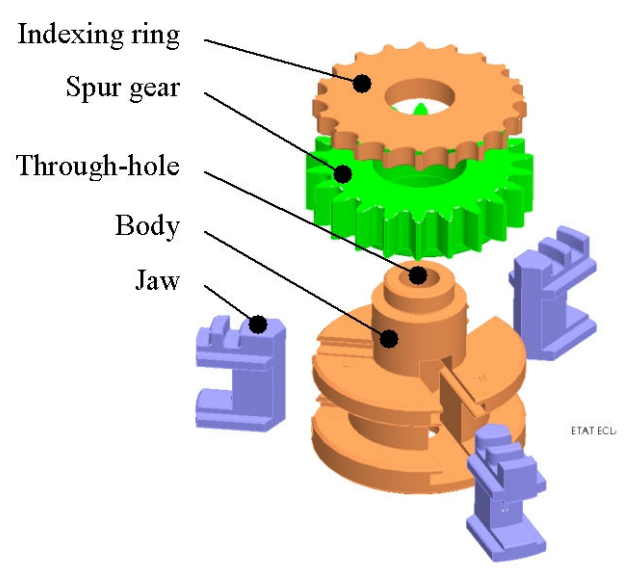

Figure 4. EXPLODED VIEW OF A CHUCK.

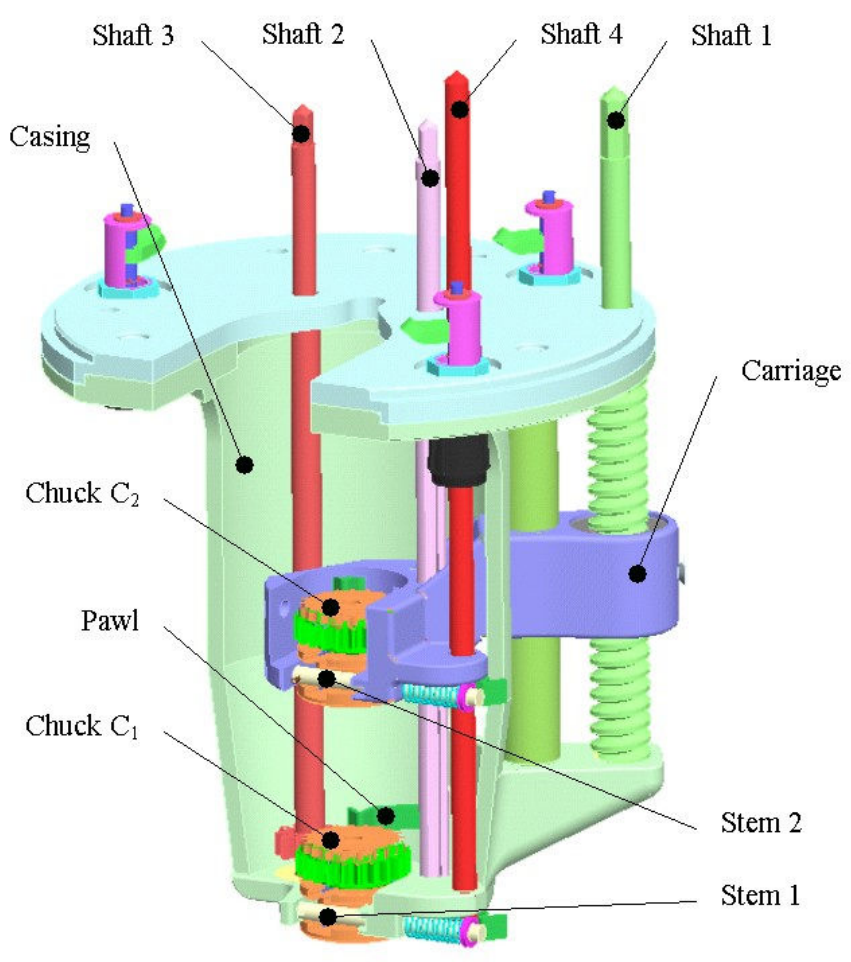

Figure 5. INSERTION MECHANISM.

For each chuck, a pawl system controls the rotation of the indexing ring so that the chuck self-rotation is blocked during a needle release. On the opposite, the chuck and the needle can turn simultaneously during a needle tightening if the applied torque is beyond a threshold defined by the pawl system design.

The two chucks are locked in position using two stems (Figure 5). These stems are pushed in release position by two com- pression springs so that a rotation of the shaft 4 induces the extraction of the chucks.

Driving Mechanism Design. The driving mechanism is composed of two piezoelectric ultrasonic motors $M_{1}$ and $M_{2}$ (Figure 6). The shaft 1 is actuated by the motor $M_{1}$ for carriage translation. The motor $M_{2}$ drives simultaneously in rotation the shafts 2 and 3 with opposite directions, thanks to a special gear arrangement. The rotation of only one actuator operates therefore the simultaneous tightening of one chuck (for example $C_{1}$ connected to shaft 3$)$ and the loosening of the other $\left(C_{2}\right.$ connected to shaft 2).

A spring-pulled locking blade is maintained armed by a controlled holding electromagnet. In case of power failure, the holding effort vanishes and the locking blade swivels of a fraction of turn. In this way, it drives the shaft 4 in rotation which itself causes the release of the stems 1 and 2 .

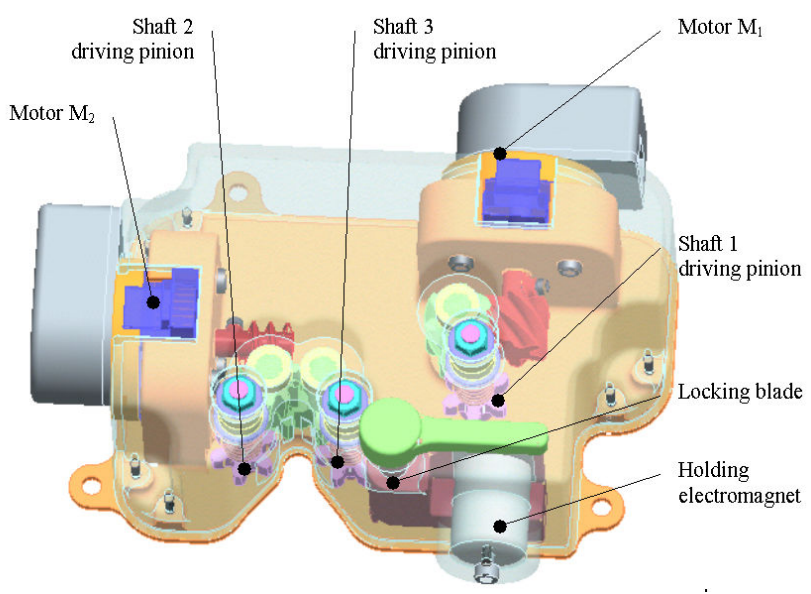

Figure 6. DRIVING MECHANISM.

\section{Device Kinematics}

Needle Translation. To perform the large needed needle translation in the limited available volume, the translational movement is split into a sum of elementary translations. These elementary displacements are obtained using translation of the carriage and alternate tightening of the chucks (figure 7).

Initially, the carriage is in its upper position and the chuck $C_{2}$ is tightened on the needle. The needle guidance is ensured by having the jaws of the lower chuck $C_{1}$ in loose contact with the needle (figure 7-a).

The insertion movement cycle is then divided into four steps:

1. The carriage is translated to its lower position (Figure 7-b). 


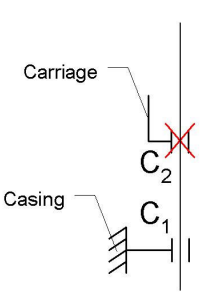

(a)

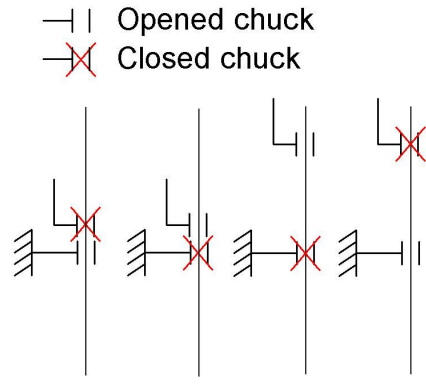

(b) (c)

(d)
Figure 7. INSERTION CYCLE.

2. The chuck $C_{2}$ is loosened while $C_{1}$ is tightened on the needle (Figure 7-c).

3. The carriage is translated back while the needle position remains constant due to its grasp with the chuck $C_{1}$ (Figure 7d).

4. The chuck $C_{2}$ on the carriage tightens whereas the lower prehensor $C_{1}$ releases the needle (Figure 7-e).

This cycle is iterated until the needle tip is at the desired position. For needle extraction, the movement is similar except that the carriage stands initially in its lower position.

Needle Rotation. As previously presented, the pawl systems installed on the chucks limit the tightening torque applied to the needle. Beyond a given torque threshold, the tightened chuck can rotate with the needle providing in this manner a security against overloading.

It should therefore be noted that the single motor $M_{2}$ not only performs the needle tightening/release but then also allows the practitioner to rotate the needle about its axis. As a result, the proposed system features a needle self-rotation capability useful for the surgeon to modify the orientation of the bevel without an additional actuator.

\section{Insertion Force Measurement}

The interface (Figure 3 ) is connected to the platform of the needle positioning robot by three 1-DOF bidirectional force sensors symmetrically placed on a circle of radius $r$ drawn around the needle axis (Figure 8). These sensors located at points $\left(A_{1}, A_{2}, A_{3}\right)$ are miniature tension/compression load cells. Vector $\vec{z}$ indicates the needle axis which is different from the direction of the gravity vector $\overrightarrow{z_{0}}$ (not represented on Figure 8 ). The entry point $B$ on the patient's skin is located at a distance $d$ from the plane $\left(A_{1}, A_{2}, A_{3}\right)$. We call $\mathcal{N}$ the system composed of the needle insertion mechanism, the needle and the three force sensors. The external actions on $\mathcal{N}$ are the force and torque exerted by the skin $S$ on the needle, the action of gravity and the action of the robot platform on the force sensors. Each sensor enables to measure the $z$-component $f_{i}$ of the action of the robot platform at point $A_{i}$. After deriving the conditions for system $\mathcal{N}$ equilibrium, the $z$-component of the effort $\overrightarrow{F_{\mathcal{S} \rightarrow \mathcal{N}}}$ exerted by the skin on the needle can be computed from the force measurements $f_{1}, f_{2}$ and $f_{3}$ :

$$
\overrightarrow{F_{\mathcal{S} \rightarrow \mathfrak{N}}} \cdot \vec{z}=M g \overrightarrow{z_{0}} \cdot \vec{z}-f_{1}-f_{2}-f_{3}
$$

where $M$ and $-g \overrightarrow{z_{0}}$ denote the mass of the insertion mechanism attached to the platform of the robot and the gravity acceleration vector. The platform orientation remains constant during the insertion so that $M g \overrightarrow{z_{0}} \cdot \vec{z}$ is a measurement offset easily identified before the needle penetration.

The special arrangement of the 1-axis bidirectional sensors enables us also to calculate two components of the torque exerted by the skin on the needle, expressed in $O$ :

$$
\begin{aligned}
& \vec{T}_{O, S \rightarrow \mathcal{N}} \cdot \vec{x}=\operatorname{Mg}\left(\overrightarrow{O G} \times \overrightarrow{z_{0}}\right) \cdot \vec{x}-\frac{r}{2} f_{2}-\frac{r \sqrt{3}}{2} f_{3} \\
& \vec{T}_{O, S \rightarrow \mathcal{N}} \cdot \vec{y}=\operatorname{Mg}\left(\overrightarrow{O G} \times \overrightarrow{z_{0}}\right) \cdot \vec{y}+r f_{1}-\frac{r \sqrt{3}}{2} f_{2}-\frac{r}{2} f_{3}
\end{aligned}
$$

where $G$ denotes the center of mass of the insertion mechanism and the $\times$ symbol stands for the vector product. These informations on the torque applied to the needle can be useful for control purposes to detect lateral flexure under needle insertion loading.

\section{Medical Requirements}

With the proposed design, the robotized insertion device exhibits several safety properties. First, the grasping of the needle and its displacement are completely decoupled from a kinematic point-of-view and operated by non-backdrivable transmissions. The control of the system is hence simplified and the needle tip position is not influenced by the interaction with the environment. Furthermore, the use of ultrasonic motors enhances safety in the event of a power failure since the configuration of the needle driver remains unchanged. Finally, the chucks linked to the needle can be extracted at any time during the needle insertion or extraction, thanks to the holding electromagnet located on the driving mechanism. The robotic system can therefore be disengaged in a few seconds if it is necessary for medical reasons.

Concerning the sterility requirement, it should be noted that the proposed needle insertion device has two main parts. The active part of the system comprising the force sensors and the driving mechanism is attached to the platform of the positioning robot. The robot and the active part of the needle insertion device can be wrapped in a sterile bag whereas the passive part corresponding to the needle insertion mechanism can be easily detached and sterilized. The chucks constructed out of polycarbonate can also be sterilized and used as disposable units. 


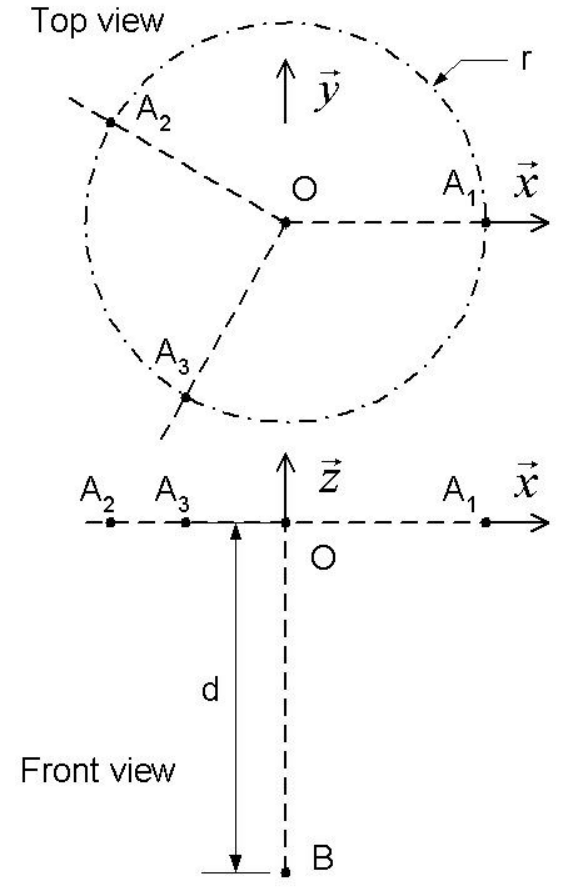

Figure 8. SCHEMATIC REPRESENTATION OF FORCE SENSORS LOCATION.

\section{CURRENT DEVELOPMENT AND PRELIMINARY EX- PERIMENTS}

In this section we present the current state of development of the needle insertion device. A first prototype has been produced to achieve some preliminary experiments concerning the CT-scan compatibility and the tightening capability of the chucks.

\section{Current Development}

The first release of a prototype was constructed to validate the presented design. Most of the parts were obtained using a rapid prototyping system except the threaded shaft 1 that was machined with conventional means (Figure 9). In the same way, since the chucks physical properties needed to be close to the final-product ones a mold was constructed using rapid tooling to produce polycarbonate chucks (Figure 10). Due to their small size, mechanisms such as the pawl systems and the quick needle release feature are still in development.

\section{Needle Tightening Capability}

Initially we expected to perform insertion forces up to $20 \mathrm{~N}$ [7]. However, previous experiments [11] showed that this constraint for the robotized insertion device was somewhat overestimated. Experiments have been achieved to validate the currently developed prototype behavior, and especially the absence of nee-

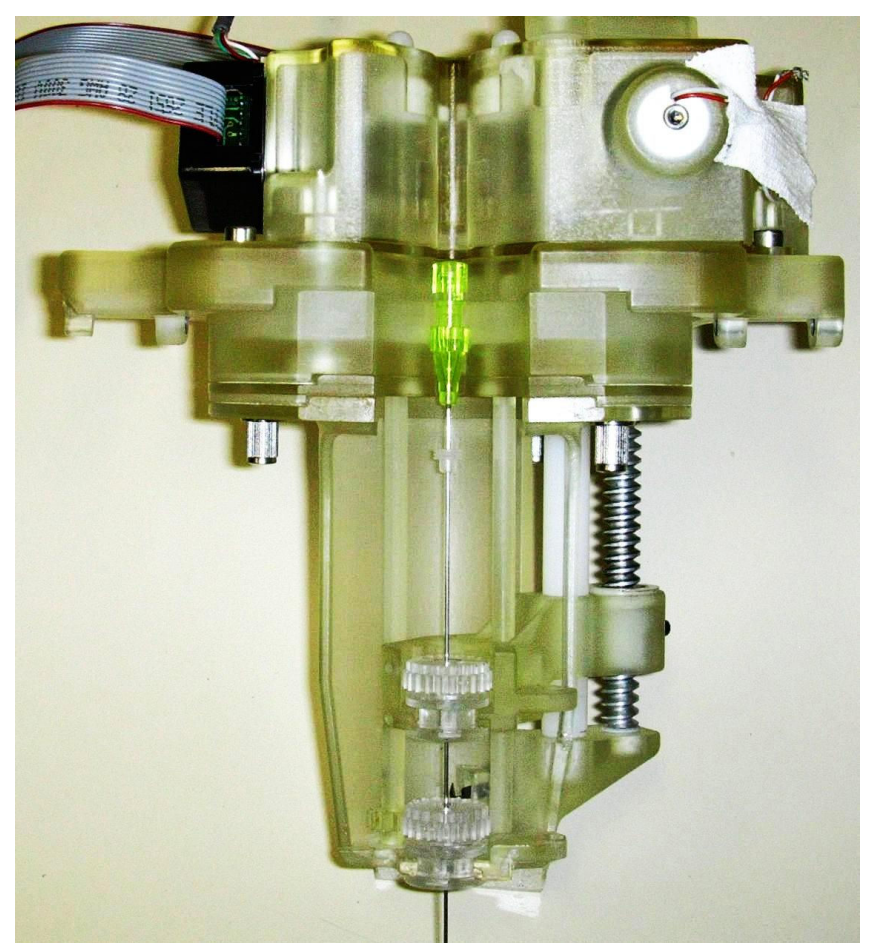

Figure 9. FABRICATED PROTOTYPE OF THE INSERTION DEVICE.

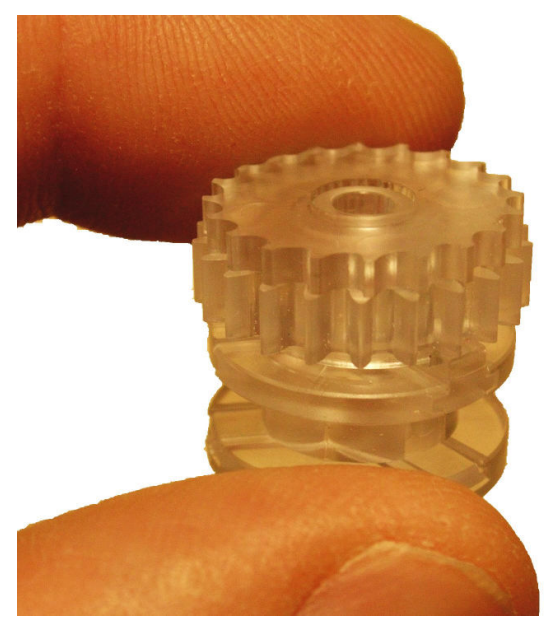

Figure 10. FABRICATED PROTOTYPE OF A CHUCK.

dle sliding in the chucks.

The experimental set-up is composed of the insertion device, a needle, an ATI Nano-17 6-axis force sensor, a PC for data acquisition, a spring-pulled cart sliding on two columns and a 6-DOF robot FANUC LR-200i (Figure 11). The needle and the insertion device are linked to the robot, and displaced at a constant speed of $1 \mathrm{~mm} / \mathrm{s}$.

Thanks to the spring-pulled cart, the position control of the 


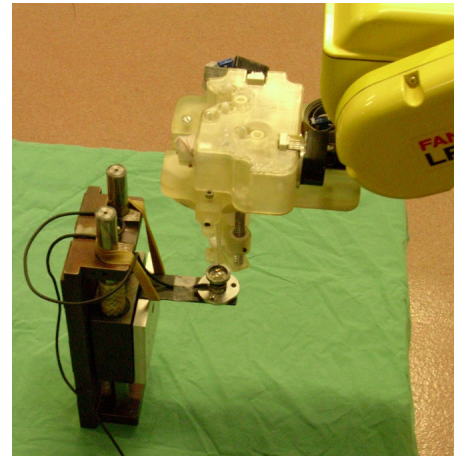

(a) Global View

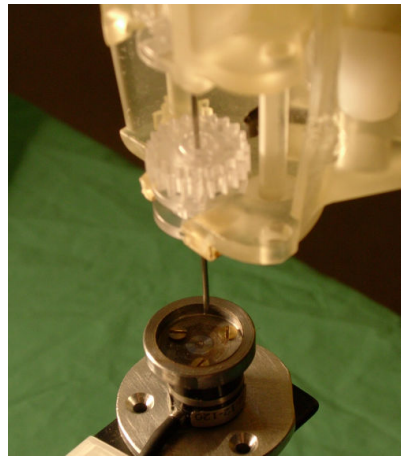

(b) Close-Up
Figure 11. EXPERIMENTAL SETUP FOR NEEDLE INSERTION EFFORT CAPABILITY.

needle tip achieved with the robot must be equivalent to a force control of the effort applied on the needle tip. Using a velocitycontrolled needle displacement, the effort measured with the force sensor in contact with the needle tip must therefore vary linearly.

The results of the experiments are given on Figure 12, where discontinuities in the force/displacement relationship are due to sliding in the chuck. No needle sliding is observed for an effort

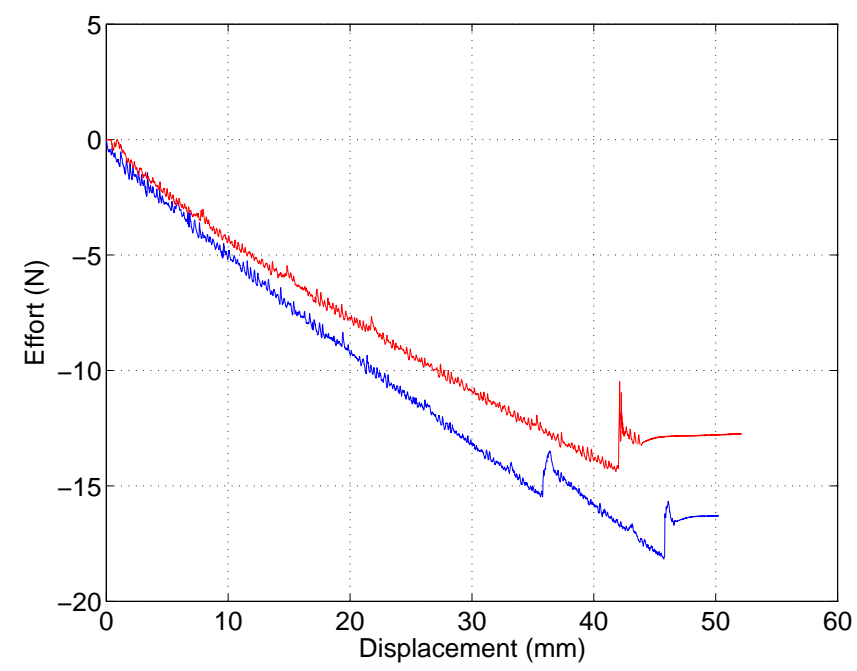

Figure 12. EFFORT MEASUREMENTS DURING THE NEEDLE DISPLACEMENT (UPPER CURVE: $0.72 \mathrm{~mm}$ NEEDLE, LOWER CURVE: $1.27 \mathrm{~mm}$ NEEDLE) - NEGATIVE VALUES ARE DUE TO THE COMPRESSION EFFORT OF THE NEEDLE TIP ON THE FORCE SENSOR.

below $10 \mathrm{~N}$ for the two tested needles of $1.27 \mathrm{~mm}$ and $0.72 \mathrm{~mm}$ diameters. The maximum transmitted efforts are equal to $15.5 \mathrm{~N}$ and $14.5 \mathrm{~N}$ for respectively the $1.27 \mathrm{~mm}$ and $0.72 \mathrm{~mm}$ needles.
The deviation between the two curves is quite certainly due to the flexure of the needles, which does not influence the experimental results. This first evaluation of the needle tightening capability of the insertion device tends to validate the proposed design.

\section{CT-Scan Compatibility}

Some experiments have been conducted at the University Hospitals of Strasbourg to perform trials on a phantom. Figure 13 shows a DICOM image acquired from the CT-scan with the needle positioning robot equipped with the insertion device. During this experiment the overall system demonstrated a correct radiolucency. Almost all the needle barrel is visible and very few artifacts in the CT-scan image are due to the insertion device itself.

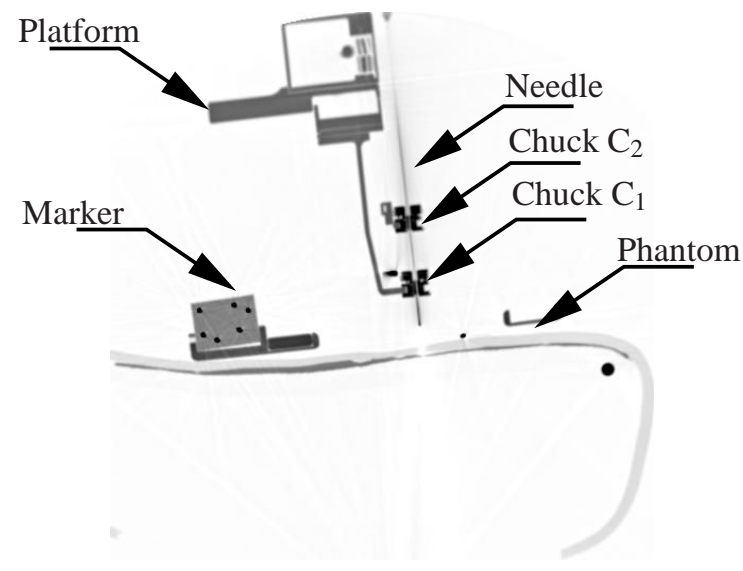

Figure 13. CT-SCAN IMAGE OF THE ROBOTIC SYSTEM.

\section{CONCLUSION}

In this paper, a new needle insertion device has been proposed. This device is included in a robotic system dedicated to gesture guidance in percutaneous procedures.

A grasp/release scheme has been proposed to accommodate various size of needles and to allow force sensors integration for haptic feedback. The specific kinematics ensures the device compactness required for a use in a CT-scan without introducing redundant actuation since two motors control the two required DOF. With the proposed design, the needle displacements are controlled in a safe way and quite high insertion forces are possible. The system architecture facilitates its sterilization and the quick disengagement of the robotic system if required for medical reasons. 
The first release of a prototype has been issued and tested during preliminary experiments. Further evaluations will be performed on a bench and in a CT-scan to validate the design. The development of the robotic system will then include the design of a specific haptic interface to interact with the surgeon.

ACKNOWLEDGMENT The authors wish to thank the Alsace Region and the CNRS-ROBEA program for the financial support of this research work.

\section{REFERENCES}

[1] Gangi, A., and Dietemann, J.-L., 1994. Tomodensimétrie Interventionnelle. Editions Vigot, PARIS.

[2] Stoianovici, D., Whitcomb, L. L., Anderson, J. H., Taylor, R. H., and Kavoussi, L. R., 1998. "A modular surgical robotic system for image guided percutaneous procedures". In Proceedings of the 1998 International Conference on Medical Image Computing and Computer-Assisted Intervention, MICCAI'98.

[3] Navab, N., Bascle, B., Loser, M. H., Geiger, B., and Taylor, R. H., 2000. "Visual servoing for automatic and uncalibrated needle placement for percutaneous procedures". In Proceedings of the IEEE International Conference on Computer Vision and Pattern Recognition, Vol. 2, CVPR 2000, pp. 2327-2334.

[4] Cleary, K., Freedman, M., Clifford, M., Lindisch, D., Onda, S., and Jiang, L., 2001. "Image-guided robotic delivery system for precise placement of therapeutic agents". Journal of Controlled Release, 74, pp. 363-368.

[5] Lavallee, S., Troccaz, J., Gaborit, L., Cinquin, P., Benabid, A., and Hoffmann, D., 1996. Computer Integrated Surgery. MIT Press, ch. Image-Guided Operating Robot: A Clinical Application in Stereotactic Surgery, pp. 343-352.

[6] Hong, J., Dohi, T., Hasizume, M., Konishi, K., and Hata, N., 2002. "A motion adaptable needle placement instrument based on tumor specific ultrasonic image segmentation". In Proceedings of the 2002 Medical Image Computing and Computer-Assisted Intervention Conference, MICCAI'02.

[7] Maurin, B., Piccin, O., Bayle, B., Gangloff, J., de Mathelin, M., Soler, L., and Gangi, A., 2004. "A new robotic system for CT-guided percutaneous procedures with haptic feedback". In Proceedings of the 2004 Computer Assisted Radiology and Surgery Congress, CARS'04.

[8] Maurin, B., Piccin, O., Bayle, B., Gangloff, J., and de Mathelin, M., 2004. "A parallel 5 dof positioner for semi-spherical workspaces". In Proceedings of the 2004 ASME Design Engineering Technical Conferences, DETC'04.

[9] Maurin, B., Doignon, C., de Mathelin, M., and Gangi, A., 2003. "Pose reconstruction with an uncalibrated computed tomography imaging device". In Proceedings of the IEEE International Conference on Computer Vision and Pattern Recognition, Vol. 1, CVPR 2003, pp. 455-460.

[10] Stoianovici, D., Cadeddu, J. A., R. D. Demaree, H. A. B., Taylor, R. H., Whitcomb, L. L., and Kavoussi, L. R., 1997. "A novel mechanical transmission applied to percutaneous renal access". Proceedings of the ASME Dynamic Systems and Control Division, DSC-Vol. 61, pp. 401-406.

[11] Maurin, B., Barbe, L., Bayle, B., Zanne, P., Gangloff, J., de Mathelin, M., Gangi, A., and Forgionne, A., 2004. "In vivo study of forces during needle insertions". In Proceedings of the 2004 Medical Robotics, Navigation and Visualisation Scientific Workshop, MRNV'04. 